Pacific Journal of Mathematic 


\section{ON COMPACT UNITHETIC SEMIGROUPS}

\section{John A. Hildebrant}

A topological semigroup is a Hausdorff space $S$ together with a continuous, associative multiplication. If each element of $S$ has unique roots in $S$ of each positive integral order, then $S$ is said to be uniquely divisible. The closure of the set of positive rational powers of an element $x$ in a compact uniquely divisible semigroup $S$ is a commutative clan (compact connected semigroup with identity) called the unithetic semigroup generated by $x$.

The purpose of this paper is to discuss the structure of compact unithetic semigroups. It is established that if the cartesian product of two semigroups is unithetic, then both factors are unithetic, and at least one factor is a group.

A partial converse is presented. If $S$ is a compact first countable unithetic semigroup, and $G$ is a finite dimensional compact unithetlc group, then $G \times S$ is a unithetic semigroup. These results are used to give the precise of a unithetic semigroup with zero whose maximal group containing the identity is finite dimensional. A complete converse to the first result is not known. In particular, the question as to whether one or both of the conditions that $S$ be first countable and $G$ be finite dimensional can be omitted is open.

Throughout this paper $R$ denotes the set of all positive rational numbers, and $N$ denotes the set of all positive integers.

A semigroup $S$ is said to be uniquely divisible if each element of $S$ has a unique root of each positive integral order. If $S$ is uniquely divisible, $x \in S$, and $n \in N$, then $x^{1 / n}$ denotes the unique $n$ th. root of $x$ in $S$. If $r \in R, r=m / n, m, n \in N$, define $x^{r}=\left(x^{1 / n}\right)^{m}$. It is not difficult to show that $x^{r}$ is unique and independent of the choice of $m$ and $n$. Define $[x]=\left\{x^{r}: r \in R\right\}^{*}$ (closure in $S$ ). If $S=[y]$ for some $y \in S$, then $S$ is said to be unithetic and $y$ a unithetic generator of $S$.

ExAMPLE. Let $I=[0,1]$ be the unit interval under usual real multiplication. Then $I$ is unithetic and is generated by any $x \in I$ such that $0<x<1$. A semigroup which is iseomorphic (topologically isomorphic) to $I$ is called a $U$-semigroup.

Note that although $I$ is unithetic, $I \times I$ is not unithetic. However, $I \times I$ is uniquely divisible. Indeed, the cartesian product of two uniquely divisible semigroups is uniquely divisible. One might ask "Under what conditions is the cartesian product of two unithetic semigroups unithetic?" This question is partially answered in $\$ 4$. 
The study of unithetic semigroups is partially motivated by the fact that each clan determines an irreducible uniquely divisible clan. This is established in the following theorem sequence:

Lemma 1.1 Let $A$ be a commutative divisible clan. For each $n \in N$, let $S_{n}=A$. For $m>n$ in $N$, define $f_{m n}: S_{m} \rightarrow S_{n}$ by $f_{m n}(z)=$ $z^{\phi: m, n)}$, where $\phi(m, n)=m ! / n !$. Let $S=\lim \left(S_{n}, f_{m n}, N\right)$. Then $S$ is a

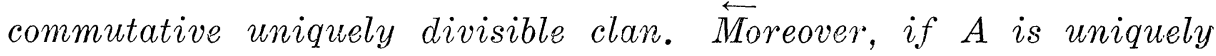
divisible, then $S$ is iseomorphic to $A$.

The proof of Lemma 1.1 is straight forward and will not be presented here. Although it is stated for clans, it holds for compact semigroups.

The definition of an irreducible clan (semigroup) is given in [5]. Note that any irreducible clan is commutative [6].

\section{Lemma 1.2. An irreducible clan $A$ is divisible.}

Proof. Let $n \in N$. Define $g_{n}: A \rightarrow A$ by $g^{n}(z)=z^{n}, z \in A$. Then,. since $A$ is a commutative semigroup, $g_{n}$ is a continuous homomorphism. Thus $g_{n}(A)$ is a subclan of $A$ which contains the identity of $A$ and. meets the kernel (minimal ideal) of $A$. Since $A$ is irreducible, $g_{n}(A)=$ $A$. Thus $A$ is divisible.

Lemma 1.3. Let $S$ be a clan and $T$ an irreducible subclan. (which contains the identity and meets the kernel of $S$ ). If $S$ is iseomorphic to $T$, then $T=S$.

Proof. Let $g: S \rightarrow T$ be an iseomorphism. Suppose $T \neq S$. Then, since $g$ is one-to-one, $g(T)$ is a proper subset of $T$. But $g(T)$ is a. subclan of $T$. This contradicts the fact that $T$ is irreducible. Hence $T=S$.

THEOREM 1.4. Each clan $S$ determines an irreducible uniquely divisible clan $S_{0}$.

Proof. The clan $S$ contains an irreducible subclan $A$ which is commutative [6]. By Lemma 1.2, $A$ is divisible. Let $A_{n}=A$ for each $n \in N$ and apply Lemma 1.1 to obtain $S_{0}=\lim A_{n}$. Then $S_{0}$ is a commutative uniquely divisible clan.

Now $S_{0}$ contains an irreducible subclan $T$ (which contains the identity and meets the kernel af $S_{0}$ ) [6]. Let $\pi_{n}, n \in N$, denote the projection of $S_{0}$ onto $A_{n}$. If $\pi_{n}(T) \neq A_{n}$ for some $n \in N$, then $\tau_{n}(T)$ is a proper subclan of $A_{n}$ which contains the identity of 
$A_{n}$ and meets the kernel of $A_{n}$. This contradicts the fact that $A_{n}$ is irreducible. Hence $\pi_{n}(T)=A_{n}$ for each $n \in N$. Now $T$ is iseomorphic to $\lim \pi_{n}(T)=\lim A_{n}=S_{0}$. Thus, $S_{0}$ and $T$ are iseomorphic. By Lemma $1.3, S_{0} \leftrightarrows T$, and hence $S_{0}$ is irreducible.

Corollary 1.5. Let $S$ be a clan containing exactly two idempotents. Then $S$ determines a compact unithetic semigroup $S_{0}$.

\section{Unithetic groups.}

THEOREM 2.1. Let $G$ be a compact uniquely divisible abelian group. These are equivalent:

(i) $G$ is monothetic;

(ii) $G$ is unithetic;

(iii) $G$ is separable.

Proof. Since $G$ is divisible and compact, $G$ is connected [3, p. 385].

(i) implies (ii). Suppose $G$ is monothetic. Then there exists $g \in G$ such that $G=\left\{g, g^{2}, \cdots\right\}^{*}$. Thus $G=\left\{g, g^{2}, \cdots\right\}^{*} \subset[g] \subset G$, and hence $G=[g]$.

(ii) implies (iii). Suppose $G$ is unithetic. Then $G=[g]$ for some $g \in G$. Hence $\left\{g^{r}: r \in R\right\}$ is dense in $G$, and thus $G$ is separable.

(iii) implies (i). Suppose $G$ is separable. Then, since $G$ is connected, $G$ is monothetic [2].

Notation. Let $\Sigma$ denote the $a$-adic solenoid with $a=(2,3,4, \cdots)$ [3, p. 114].

Let $\Sigma^{\wedge}=\prod_{\alpha \varepsilon^{\wedge}} \Sigma_{\alpha}$, where $\Sigma_{\alpha}=\Sigma$ for each $\alpha \varepsilon^{\wedge}$.

THeOREM 2.2 Let $G$ be a nondegenerate compact uniquely divisible abelian group. Then $G=\Sigma^{\wedge}$ for some^ .

Proof. Since $G$ is uniquely divisible, it is both divisible and torsion-free. The result now follows from [3, p. 406].

THEOREM 2.3. Let $G$ be a compact group. Then $G$ is unithetic if and only if $G=\Sigma^{\wedge}$ and card $^{\wedge} \leqq c$.

Proof. Suppose $G$ is unithetic. Then $G$ is a uniquely divisible abelian group. Hence, by Theorem 2.2, $G=\Sigma^{\wedge}$ for some ^. By Theorem 2.1, $G$ is separable. Thus card ${ }^{\wedge} \leqq c$ [10].

Suppose $G=\Sigma^{\wedge}$ and card ${ }^{\wedge} \leqq c$. Then $G$ is separable [10]. Hence, by Theorem 2.1, $G$ is unithetic. 
COROLLARY 2.4. Any element of $\Sigma$ which is not the identity is a unithetic generator of $\Sigma$.

Lemma 2.5. Let $\left\{r_{n}\right\}$ be a sequence in $R$ which converges to 0 . Then there exist $g, g_{0} \in \Sigma, g_{0} \neq 1$, such that $\left\{g^{r_{n}}\right\}$ clusters to $g_{0}$.

Proof. Let $N$ denote the set of all positive integers and $C$ the circle group (the boundary of the complex unit disk under multiplication). For each $n \in N$, let $C_{n}=C$. Define $f_{m n}: C_{m} \rightarrow C_{n}$ for $m>n$, by $f_{m n}(z)=z^{\phi(m, n)}$, where $\phi(m, n)=m ! / n !$. Then $\Sigma=\lim \left(C_{n}, f_{m n}, N\right)$.

Let $L$ denote the closed left half of $C$, i.e., $L=\left\{e^{i \theta}: \cos \theta \leqq 0\right\}$. We will construct, by induction, an element $g=\left(g_{1}, g_{2}, \cdots\right)$ in $\Sigma$ such that $\left\{r_{n}\right\}$ has a subsequence $\left\{r_{n}\right\}$ such that each first coordinate of $g^{r_{n_{j}}}$ lies in $L$. This will be sufficient to insure that $\left\{g^{r} n_{j}\right\}$ does not converge to the identity $(1,1,1, \cdots)$ of $\Sigma$. Thus $\left\{g^{r_{n}}\right\}$ (hence $\left\{g^{r}{ }^{r}\right\}$ ) will have a subsequence converging to some $g_{0} \neq 1$ in $\Sigma$.

Note that if $r=a / b$, where $a$ and $b$ are relatively prime positive integers, and $\left(x_{1}, x_{2}, \cdots\right)$ is an element of $\Sigma$, then $\left(x_{1}, x_{2}, \cdots\right)^{r}=$ $\left(x_{b}^{r b !}, \cdots\right)$.

For each $r_{n}$ in $\left\{r_{n}\right\}$ let $m_{n}$ be the least positive integer such that $M_{n}=m_{n} ! r_{n}$ is an integer. We may assume that $\left\{r_{n}\right\}$ is such that $m_{1}>2$ and that $m_{n-1}<m_{n}$ for each $n$, since $\left\{r_{n}\right\}$ will have a subsequence satisfying these conditions.

Let $r_{n}=a_{n} / b_{n}$, where $a_{n}$ and $b_{n}$ are relatively prime positive integers for each $n$.

Let $g_{1}=g_{2}=\cdots=g_{m_{1}}=1$. We want to define $g=\left(g_{1}, g_{2}, \cdots\right)$ so that:

(i) $g_{n}^{n}=g_{n-1}$ for each $n$, i.e., $g \in \Sigma$, and

(ii) $g_{m_{n}}^{M_{n}} \in L$ for each $n \geqq 2$.

Note that having defined $g_{n-1}$ we can always find $z \in C$ such that $z^{n}=g_{n-1}$ by selecting $z$ to be one of the $n$ th. roots of $g_{n-1}$. However, the manner in which $z$ is chosen when $n=m_{n}-1$ will be more specific, so that (ii) can be satisfied.

Having defined $g_{m_{n}}$, define $g_{m_{n}+1}, \cdots, g_{m_{n+1}-1}$ just to satisfy condition (i).

We now define $g_{m_{n}}$ by induction. Suppose that $g_{m_{n}-1}=e^{i \theta}$, where $0 \leqq \theta<2 \pi$. Let $\beta=M_{n} / m_{n}$. Then $\beta \in R \backslash N$, by the way in which $m_{n}$ is defined.

Suppose $2^{p} \beta \in N$ for some $p \in N$. Let $p_{0}=\min .\left\{p \in N: 2^{p} \beta \in N\right\}$. Then $2^{p_{0}} \beta$ is odd and hence $\cos \left(2^{p_{0}} \beta \pi\right)+\sin \left(2^{p_{0}} \beta \pi\right)=-1 \leqq 0$.

Suppose $2^{p} \beta \notin N$ for all $p \in N$. Then, using the dyadic expansion of $\beta$, one can show that there exists $p_{0} \in N$ such that

$$
\cos \left(2^{p_{0}} \beta \pi\right)+\sin \left(2^{p_{0}} \beta \pi\right) \leqq 0 .
$$

Thus, in either case, we have 


$$
\begin{aligned}
\cos \left(\theta \beta+2^{p_{0}} \beta \pi\right) & =\cos (\theta \beta) \cos \left(2^{p_{0}} \beta \pi\right)-\sin (\theta \beta) \sin \left(2^{p_{0}} \beta \pi\right) \\
& \leqq \cos \left(2^{p_{0}} \beta \pi\right)+\sin \left(2^{p_{0}} \beta \pi\right) \leqq 0 .
\end{aligned}
$$

Let $g_{m_{n}}=e^{i\left[\left(\theta / m_{n}\right)+\left(2^{p} 0_{\pi}\right) / m_{n}\right]}$. Then

$$
g_{m_{n}}^{m_{n}}=e^{i\left(\theta+2^{p} 0_{\pi}\right)}=e^{i \theta}=g_{m_{n-1}} \text {. }
$$

Moreover, $g_{m_{n}}^{M_{n}}=e^{i\left(\theta \beta+2^{p} 0_{\beta \pi)}\right.}$, which is in $L$. Thus the required conditions are satisfied.

Let $g=\left(g_{1}, g_{2}, \cdots\right)$. Then $g^{r_{n}}=\left(g_{b_{n} n^{b} n^{1}}^{r}, \cdots\right)=\left(g_{m_{n}}^{M_{n}}, \cdots\right)$ and $g_{m_{n}}^{M_{n}} \in L$ for each $n$. This completes the proof of the lemma.

3. Preliminary results. Throughout this section $S=[x]$ denotes a compact unithetic semigroup which is not a group. The results in Theorem 3.1 can be obtained as a consequence of $[4, \mathrm{p} .275]$ or by using the techniques and results in [7], [8], and [9].

For a net $\left\{x_{\alpha}\right\}$ in $S$, we use $x_{\alpha} \stackrel{e}{\longrightarrow} x$ to denote the fact that $\left\{x_{\alpha}\right\}$ converges to $x$, and $x_{\alpha} \stackrel{f}{\longrightarrow} x$ to denote the fact that $\left\{x_{\alpha}\right\}$ clusters to $x$.

THEOREM 3.1. The semigroup $S$ is a commutative clan containing exactly two idempotents such that:

(i) The kernel $K(x)$ of $S$ is a unithetic group generated by ex, where $e$ is the identity of $K(x)$.

(ii) The maximal subgroup $H(x)$ containing the identity 1 of $S$ is a unithetic group.

(iii) There is a continuous one-to-one homomorphism $\sigma$ from the additive nonnegative real numbers $\bar{R}$ into $S$ such that $S=H(x)\left(\sigma(\bar{R})^{*}\right)$. The kernel of $\sigma(\bar{R})^{*}$ is $\sigma(\bar{R})^{*} \backslash \sigma(\bar{R})$ and is contained in $K(x)$. If $\sigma(\alpha)=\sigma(\beta) g$, for $\alpha, \beta \in \bar{R}$ and $g \in H(x)$, then $\alpha=\beta$ and $g=1$. Moreover, if $\alpha \in \bar{R} \backslash\{0\}$, then $\sigma(\bar{R})^{*}=[\sigma(\alpha)]$.

LeMma 3.2. Let $\left\{r_{\alpha}\right\}$ be a net in $R$.

(i) If $r_{\alpha} \stackrel{e}{\longrightarrow} 0$ and $x^{r \alpha} \stackrel{f}{\longrightarrow} z$, then $z \in H(x)$.

(ii) If $x^{r_{\alpha}} \stackrel{e}{\longrightarrow} z$ and $z \in H(x)$, then $r_{\alpha} \stackrel{f}{\longrightarrow} 0$.

Proof. For $s \in R$, let $H(x, s)=\left\{x^{r}: r \in R, r<s\right\}^{*}$. Then $H(x)=$ ก $\{H(x, s): s \in R\}$ (See [8]).

(i) Let $s \in R$. Then there exists $\alpha_{0}$ such that $r_{\alpha}<s$ for all $\alpha>\alpha_{0}$. Thus $x^{r} \in H(x, s)$ for all $\alpha>\alpha_{0}$. Since $H(x, s)$ is closed and $x^{r_{\alpha}} \stackrel{f}{\longrightarrow} z, z \in H(x, s)$. Hence $z \in H(x)$.

(ii) Suppose there exists $r \in R$ such that $r_{\alpha}>r$ eventually. Then

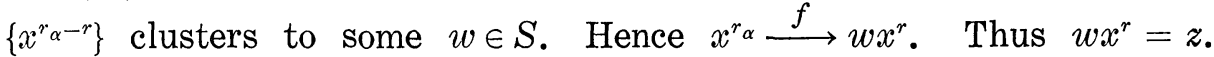
Therefore, $w^{1 / r} x=z^{1 / r}$, and $z^{1 / r} \in H(x)$. Since $S \backslash H(x)$ is an ideal, $x \in H(x)$. 
This implies that $S$ is a group, which contradicts the assumption that $S$ is not a group. Hence $r_{\alpha} \stackrel{f}{\longrightarrow} 0$.

TheOREM 3.3. The function $\Psi: R \rightarrow S$ defined by $\Psi(r)=x^{r}, r \in R$, is continuous if and only if $H(x)=\{1\}$.

Proof. If $H(x)=\{1\}$, then the fact that $\Psi$ is continuous follows from Lemma 3.2 (i).

Suppose $H(x) \neq\{1\}$. Let $p \in H(x), p \neq 1$. Let $\left\{s_{\alpha}\right\}$ be a net in $R$ such that $x^{s_{\alpha}} \stackrel{e}{\longrightarrow} p$. Then, by Lemma 3.2 (ii), $s_{\alpha} \stackrel{f}{\longrightarrow} 0$. Let $s \in R$. Then there exists a subnet $\left\{s_{\alpha_{\beta}}\right\}$ of $\left\{s_{\alpha}\right\}$ such that $s_{\alpha_{\beta}} \stackrel{e}{\longrightarrow} 0$ and $s_{\alpha_{\beta}}<s$ for all $\beta$. Thus $s-s_{\alpha_{\beta}} \stackrel{e}{\longrightarrow} s$. If $\Psi$ were continuous, we would have $x^{s-s_{\alpha}} \stackrel{e}{\longrightarrow} x^{s}$, and thus $x^{s}=p x^{s}$ or $x=p^{1 / s} x$. This would imply that $p=1$. Since $p \neq 1, \Psi$ is not continuous.

THEOREM 3.4. The quotient semigroup $S / K(x)$ is a compact unithetic semigroup which is iseomorphic to $(H(x) \times I) /(H(x) \times\{0\})$, where $I=[0,1]$ is a U-semigroup. A generator of $S / K(x)$ is $\phi(x)$, where $\phi: S \rightarrow S / K(x)$ is the natural map.

Proof. It is not difficult to show that $S / K(x)$ is a uniquely divisible commutative clan with zero $z=\phi(K(x))$.

Let $\phi(y) \in S / K(x), y \in S$. Then there exists a net $\left\{r_{\alpha}\right\}$ in $R$ such that $x^{r_{\alpha}} \stackrel{e}{\longrightarrow} y$. Since $\phi$ is continuous, $\phi\left(x^{r_{\alpha}}\right) \stackrel{e}{\longrightarrow} \phi(y)$. Since $\phi$ is a homomorphism $\phi\left(x^{r} \alpha\right)=\phi(x)^{r_{\alpha}}$ for each $\alpha$. Thus $\phi(y) \in[\phi(x)]$, and hence $S / K(x)=[\phi(x)]$.

Let $\sigma$ be the map of Theorem 3.1. Then $S=H(x)\left(\sigma(\bar{R})^{*}\right)$, and hence $S / K(x)=\phi(H(x)) \phi\left(\sigma(\bar{R})^{*}\right)$. Since the kernel of $\sigma(\bar{R})^{*}$ is contained in $K(x), z$ is a zero for $\phi\left(\sigma(\bar{R})^{*}\right)$.

Define $f: \phi(H(x)) \times \phi\left(\sigma(\bar{R})^{*}\right) \rightarrow S / K(x)$ by $f((a, b))=a b$. Then $f$ is a continuous homomorphism onto $S / K(x)$. Define a relation $Q$ on $\phi(H(x)) \times \phi\left(\sigma(\bar{R})^{*}\right.$ by $Q=\{(a, b),(c, d): f((a, b))=f((c, d))\}$. Then $(\phi(H(x)) \times \phi(\sigma(\bar{R}))) / Q$ is iseomorphic to $S / K(x)$.

It will be established that

$$
\left(\phi(H(x)) \times \phi\left(\sigma(\bar{R})^{*}\right) / Q=\left(\phi(H(x)) \times \phi\left(\sigma(\bar{R})^{*}\right)\right) /(\phi(H(x)) \times\{z\}) .\right.
$$

This is done by showing that $f$ is one-to-one on $\phi(H(x)) \times\left(\phi\left(\sigma(\bar{R})^{*}\right) \backslash\{z\}\right)$ (\ denotes complement).

Suppose $f\left(\left(a^{\prime}, b^{\prime}\right)\right)=f\left(\left(c^{\prime}, d^{\prime}\right)\right)$, where $a^{\prime}, c^{\prime} \in \dot{\phi}(H(x))$ and

$$
b^{\prime}, d^{\prime} \in \phi\left(\sigma(\bar{R})^{*}\right) \backslash\{z\} \text {. }
$$

Then $a^{\prime} b^{\prime}=c^{\prime} d^{\prime}$. There exist $a, c \in H(x)$ and $b, d \in \sigma(\bar{R})^{*}$ such that $a^{\prime}=$ 
$\dot{\phi}(a), b^{\prime}=\dot{\phi}(b), c^{\prime}=\phi(c)$, and $d^{\prime}=\phi(d)$. Since $b^{\prime} \neq z \neq d^{\prime}, b^{\prime}$ and $d$ are not in the kernel of $\sigma(\bar{R})^{*}$. Thus, by Theorem 3.1, $b, d \in \sigma(\bar{R})$. Now $\dot{\phi}(a b)=\dot{\phi}(a) \dot{\phi}(b)=a^{\prime} b^{\prime}=c^{\prime} d^{\prime}=\dot{\phi}(c) \phi(d)=\dot{\phi}(c d)$.

Suppose $a b \in K(x)$. Let $a^{-1}$ denote the inverse of $a$ in $H(x)$. Then $b=1 \cdot b=\left(a^{-1} a\right) b=a^{-1}(a b)$ is in $K(x)$. Thus $\phi(b)=z$. This contradicts $b^{\prime} \neq z$. Hence $a b \in S \backslash K(x)$. Similarly, $c d \in S \backslash K(x)$.

Since $\dot{\phi}$ is one-to-one on $S \backslash K(x)$ and $\dot{\phi}(a b)=\dot{\phi}(c d), a b=c d$. Let $\alpha \in \bar{R}$ and $\beta \in \bar{R}$ such that $b=\sigma(\alpha)$ and $d=\sigma(\beta)$. Then $a \sigma(\alpha)=c \sigma(\beta)$. Thus $\sigma(\alpha)=\left(a^{-1} c\right) \sigma(\beta)$ and $a^{-1} c \in H(x)$. Hence, by Theorem 3.1, $\alpha=\beta$ and $a^{-1} c=1$. Therefore, $a=c$ and $b=d$, and hence $f$ is one-to-one $\phi(H(x)) \times\left(\dot{\phi}\left(\sigma(R)^{*}\right) \backslash\{z\}\right)$. This establishes the fact $S / K(x)$ is iseomorphic to $\left(\dot{\phi}(H(x)) \times \dot{\phi}\left(\sigma(\bar{R})^{*}\right) /\left(\dot{\phi}(H(x)) \times\left\{z_{j}\right)\right.\right.$.

Since $\dot{\phi}$ is one-to-one on $S \backslash K(x), \dot{\phi}(H(x))$ is iseomorphic to $H(x)$.

Let $I=[0,1]$ under usual real multiplication. Then $I$ is a compact unithetic semigroup. Let $\sigma_{0}$ be the map of Theorem 3.1 corresponding to $I$. Since the maximal group of $I$ containing 1 is trivial, $I=\sigma_{0}(\bar{R})^{*}$. Moreover, since the kernel of $I$ is $\{0\},(0,1]=\sigma_{0}(\bar{R})$. Define $u: \phi\left(\sigma(\bar{R})^{*}\right) \rightarrow$ $I$ by $u(z)=0$ and $u(b)=\sigma_{0}(\alpha)$, where $b=\phi(\sigma(\alpha))$ for $\alpha \in \bar{R}$ if $b \neq z$. Then $u$ is an iseomorphism of $\dot{\phi}\left(\sigma(\bar{R})^{*}\right)$ onto $I$. This completes the proof of the theorem.

\section{Structure theorems.}

THEOREM 4.1. Let each of $S_{1}$ and $S_{2}$ be compact semigroups such that $S_{1} \times S_{2}$ is unithetic. Then each of $S_{1}$ and $S_{1}$ is unithetic. Moreover, either $S_{1}$ or $S_{2}$ is a group.

Proof. Let $S_{1} \times S_{2}=\left[\left(x_{1}, x_{2}\right)\right]$. Then $S_{1}=\left[x_{1}\right]$ and $S_{2}=\left[x_{2}\right]$.

Suppose that neither $S_{1}$ nor $S_{2}$ is a group. Now $\left(1, x_{2}\right) \in S_{1} \times S_{2}$. Hence there exists a net $\left\{r_{\alpha}\right\}$ in $R$ such that $x_{1}^{r_{\alpha}} \stackrel{e}{\longrightarrow} 1$ and $x_{2}^{r_{\alpha}} \stackrel{e}{\longrightarrow} x_{2}$. Since $x_{1}^{r} \stackrel{e}{\longrightarrow} 1, r_{\alpha} \stackrel{f}{\longrightarrow} 0$. Let $\left\{r_{\alpha_{\beta}}\right\}$ be a subnet such that $r_{\alpha_{\beta}} \stackrel{e}{\longrightarrow} 0$. Then $x_{2}^{r_{\alpha}} \stackrel{e}{\longrightarrow} x_{2}$. But this implies that $r_{\alpha_{\beta}} \stackrel{f}{\longrightarrow} 1$. This contradiction implies that either $S_{1}$ or $S_{2}$ is a group.

THEOREM 4.2. Let $S=[x]$ be a compact first countable unithetic semigroup, and $G$ a compact finite dimensional unithetic group. Then $G \times S$ is a unithetic semigroup.

Proof. If $S$ is a group, then the theorem follows from Theorem 2.3 , since $G=\Sigma^{\wedge}$ for some finite ^.

Suppose that $S$ is not a group. We prove that $\Sigma \times S$ is unithetic. The conclusion follows by induction.

Now $\Sigma \times S$ is a compact uniquely divisible semigroup with identity 
$(1,1)$ and maximal group $\Sigma \times H(x)$.

Let $\left\{r_{n}\right\}$ be a sequence in $R$ such that $x^{r_{n}} \stackrel{e}{\longrightarrow} 1$ and $r_{n} \stackrel{e}{\longrightarrow} 0$. Then, by Lemma 2.5, there exist $g \in \Sigma$ and $g_{0} \in \Sigma, g_{0} \neq 1$, such that $g^{r_{n}} \stackrel{f}{\longrightarrow} g_{0}$. Thus $(g, x)^{r_{n}} \stackrel{f}{\longrightarrow}\left(g_{0}, 1\right)$. Hence $\left(g_{0}, 1\right) \in H((g, x))$. Therefore, $H((g, x)) \cap[\Sigma \times\{1\}]$ is a nondegenerate compact uniquely divisible subgroup of $\Sigma \times\{1\}$. Thus, by Corollary 2.4, $H((g, x)) \cap(\Sigma \times\{1\})=$ $\Sigma \times\{1\}$, and hence $\Sigma \times\{1\} \subset[(g, x)]$.

Let $(w, y) \in \Sigma \times S$, and $x^{s_{j}} \stackrel{e}{\longrightarrow} y, s_{j} \in R$. Then $g^{s_{j}} \stackrel{f}{\longrightarrow} g_{0} \in \Sigma$. Hence $\left(g_{0}, y\right) \in[(g, x)]$. Since $\Sigma \times\{1\} \subset[(g, x)],\left(w g_{0}^{-1}\right) \in[(g, x)]$. Hence $(w, y)=\left(w g_{0}^{-1}, 1\right)\left(g_{0}, y\right)$ is in $[(g, x)]$. Thus $\Sigma \times S \subset[(g, x)]$, and hence $\Sigma \times S=[(g, x)]$. This completes the proof of the theorem.

The following theorem is a consequence of Theorem 3.4.

TheOREM 4.3. Let $S_{1}=\left[x_{1}\right]$ and $S_{2}=\left[x_{2}\right]$ be compact unithetic semigroups such that $H\left(x_{1}\right)$ is iseomorphic to $H\left(x_{2}\right)$. Then $S_{1} / K\left(x_{1}\right)$ is iseomorphic to $S_{2} / K\left(x_{2}\right)$.

EXAMPLE. Let $I=[0,1]$ be a $U$-semigroup and ${ }^{\wedge}$ a set of $n$ elements. Then, by Theorem 4.2, $\Sigma^{\wedge} \times I$ is unithetic. Moreover, $D_{n}=$ $\left(\Sigma^{\wedge} \times I\right) /\left(\Sigma^{\wedge} \times\{0\}\right)$ is a unithetic semigroup with zero whose maximal group containing the identity has dimension $n$.

COROLLARY 4.4. Let $S=[x]$ be a compact unithetic semigroup with zero such that $H(x)$ has dimension n. Then $S$ is iseomorphic to $D_{n}$.

\section{REFERENCES}

1. D. R. Brown and J. G. LaTorre, A characterization of commutative uniquely divisible semigroups, Pacific J. Math. (to appear)

2. P. R. Halmos and H. Samelson, On monothetic groups, Proc. Nat. Acad. Sci. U. S. 28 (1942), 254-258.

3. E. Hewitt and K. A. Ross, Abstract Harmonic Analysis, Academic Press Inc., New York, 1963.

4. K. H. Hofmann, Topologische Halbgruppen mit dichter submonoger Untenhalbgruppe, Math. Zeit. 74 (1960), 232-276.

5. K. H. Hofmann and P. S. Mostert, Irreducible Semigroups, Bull. Amer. Math. Soc. 70 (1964), 621-627.

6. K. H. Hofmann and P. S. Mostert, (An unpublished book manuscript, to appear). 7. R. J. Koch, On monothetic semigroups, Proc. Amer. Math. Soc. 8 (1957), 397-401.

8. Anne Lester (Hudson), Some semigroups on the two-cell, Proc. Amer. Math. Soc. 10 (1959), 648-655.

9. P. S. Mostert and A. L. Shields, One-Parameter Semigroups in a Semigroup, Trans. Amer. Math. Soc. 14 (1963), 396-400.

10. K. A. Ross and A. H. Stone, Products of separable spaces, Amer. Math. Monthly, April (1964), 713-716. 
Received April 13, 1966. This paper contains part of a doctoral dissertation written under the direction of Professor D. R. Brown while the author held a National Aeronautics and Space Administration graduate fellowship.

The University of Tennessee

Knoxville, Tennessee 



\section{PACIFIC JOURNAL OF MATHEMATICS}

\section{EDITORS}

H. SAMELSON

Stanford University

Stanford, California

J. P. JANS

University of Washington

Seattle, Washington 98105

\section{J. DugunduI}

University of Southern California Los Angeles, California 90007

RICHARD ARENS

University of California

Los Angeles, California 90024

\section{ASSOCIATE EDITORS}
E. F. BECKENBACH
B. H. NEUMANN
F. WOLF
K. YoSIDA

\section{SUPPORTING INSTITUTIONS}

\author{
UNIVERSITY OF BRITISH COLUMRIA \\ CALIFORNIA INSTITUTE OF TECHNOLOGY \\ UNIVERSITY OF CALIFORNIA \\ MONTANA STATE UNIVERSITY \\ UNIVERSITY OF NEVADA \\ NEW MEXICO STATE UNIVERSITY \\ OREGON STATE UNIVERSITY \\ UNIVERSITY OF OREGON \\ OSAKA UNIVERSITY \\ UNIVERSITY OF SOUTHERN CALIFORNIA
}

\author{
STANFORD UNIVERSITY \\ UNIVERSITY OF TOKYO \\ UNIVERSITY OF UTAH \\ WASHINGTON STATE UNIVERSITY \\ UNIVERSITY OF WASHINGTON \\ AMERICAN MATHEMATICAL SOCIETY \\ CHEVRON RESEARCH CORPORATION \\ TRW SYSTEMS \\ NAVAL ORDNANCE TEST STATION
}

Mathematical papers intended for publication in the Pacific Journal of Mathematics should be typewritten (double spaced). The first paragraph or two must be capable of being used separately as a synopsis of the entire paper. It should not contain references to the bibliography. Manuscripts may be sent to any one of the four editors. All other communications to the editors should be addressed to the managing editor, Richard Arens at the University of California, Los Angeles, California 90024 .

50 reprints per author of each article are furnished free of charge; additional copies may be obtained at cost in multiples of 50 .

The Pacific Journal of Mathematics is published monthly. Effective with Volume 16 the price per volume (3 numbers) is $\$ 8.00$; single issues, $\$ 3.00$. Special price for current issues to individual faculty members of supporting institutions and to individual members of the American Mathematical Society: $\$ 4.00$ per volume; single issues $\$ 1.50$. Back numbers are available.

Subscriptions, orders for back numbers, and changes of address should be sent to Pacific Journal of Mathematics, 103 Highland Boulevard, Berkeley 8, California.

Printed at Kokusai Bunken Insatsusha (International Academic Printing Co., Ltd.), No. 6, 2-chome, Fujimi-cho, Chiyoda-ku, Tokyo, Japan.

\section{PUBLISHED BY PACIFIC JOURNAL OF MATHEMATICS, A NON-PROFIT CORPORATION}

The Supporting Institutions listed above contribute to the cost of publication of this Journal, but they are not owners or publishers and have no responsibility for its content or policies. 


\section{Pacific Journal of Mathematics}

\section{Vol. 21, No. 2 December, 1967}

Arne P. Baartz, The measure algebra of a locally compact semigroup ..... 199

Robert F. Brown, On maps with identical fixed point sets............. 215

C. Buttin, Existence of a homotopy operator for Spencer's sequence in the analytic case ..................................... 219

Henry Werner Davis, An elementary proof that Haar measurable almost periodic functions are continuous ........................ 241

Zeev Ditzian, On asymptotic estimates for kernels of convolution transforms ...................................... 249

Robert E. Edwards, Boundedness principles and Fourier theory ......... 255

John A. Hildebrant, On compact unithetic semigroups ............... 265

Marinus A. Kaashoek and David Clark Lay, On operators whose Fredholm set is the complex plane ............................ 275

Sadao Kató, Canonical domains in several complex variables ........... 279

David Clifford Kay, The ptolemaic inequality in Hilbert geometries.... . . . 293

Joseph D. E. Konhauser, Biorthogonal polynomials suggested by the Laguerre polynomials ............................. 303

Kevin Mor McCrimmon, Macdonald's theorem with inverses .......... 315

Harry Eldon Pickett, Homomorphisms and subalgebras of multialgebras .................................... 327

Richard Dennis Sinkhorn and Paul Joseph Knopp, Concerning nonnegative matrices and doubly stochastic matrices ..............

Erling Stormer, On anti-automorphisms of von Neumann algebras ...

Miyuki Yamada, Regular semi-groups whose idempotents satisfy permutation identities .......................... 